\title{
Filaire hyperévoluée,
}

\section{parasite de la paroi intestinale d'un Coendou}

\author{
par Alain G. CHABAUD \\ Laboratoire de Zoologie (Vers) associé au C.N.R.S. (Pr A.-G. CHABaud) \\ Muséum national d'Histoire naturelle, 43, rue Cuvier, F 75231 Paris Cedex 05
}

\section{Résumé.}

Description d'une Filaire parasite de la paroi intestinale de Coendu prehensilis à Belem (Para, Brésil).

L'espèce est remarquable par son extrême gracilité (longueur de la femelle: $62,8 \mathrm{~mm}$, largeur : $50 \mu$ ) et par un cône transparent très développé à la pointe caudale du mâle. Elle est interprétée comme étant une forme hyperspécialisée du groupe des Dipetalonema, mais nécessite la création d'un genre nouveau, et est nommée Filarissima lainsoni n. g., n. sp.

\section{Summary:}

Hyperspecialised Filaria parasite of the intestinal wall of Coendu.

Description of a Filaria parasite of the intestinal wall of Coendu prehensilis from Belem (Para, Brazil).

The species is remarkable by its outstanding slenderness (lengh of the female: $62,8 \mathrm{~mm}$; width : $50 \mu$ ) and by the presence of a well developed transparent cone at the caudal extremity of the male. It is suggested by the author that the parasite is an hyperspecialised species belonging to the group Dipetalonema and makes the creation of a new genus necessary.

We name it Filarissima lainsoni n. g., n. sp.

La Filaire étudiée dans cette note est à notre connaissance le Nématode le plus gracile actuellement connu, puisque la femelle atteint une longueur de $62,8 \mathrm{~mm}$ pour une largeur de $50 \mu$. L'hôte est un Coendu prehensilis Linné capturé dans la 
région de Belem (Para-Brésil) le 16 novembre 1972. Le corps des Nématodes, invisible puisqu'il est entièrement enfoui dans la paroi digestive, essentiellement du côté péritonéal de cette paroi, est devenu apparent après section du duodenum ; certaines boucles du corps font saillie sur la tranche de la section et il a été possible d'extraire une femelle entière, une partie postérieure de mâle longue de $31,5 \mathrm{~mm}$ et quelques fragments d'autres spécimens.

\section{Description.}

Femelle holotype: Corps long de $62,8 \mathrm{~mm}$, large de $50 \mu$ au milieu du corps. Tête arrondie, large de $40 \mu$, puis rétrécie en un cou large de $35 \mu$, à $40 \mu$ de l'apex. La vue apicale n'a pu être réalisée, mais les deux cycles de quatre papilles sont visibles en vue latérale (fig. B).

L'œsophage, long de $330 \mu$, mal limité, est en voie d'atrophie (fig. A). L'intestin, assez large à sa naissance, devient rapidement très étroit (fig. A) et conserve ce diamètre réduit jusqu'au rectum. Le pore excréteur est situé à $170 \mu$ de l'apex. La place de l'anneau nerveux n'a pu être précisée.

La vulve s'ouvre un peu en arrière de la fin de l'œsophage à $390 \mu$ de l'extrémité antérieure. Elle est suivie d'une masse sphinctérienne presque sphérique de $30 \mu$ de diamètre, à l'intérieur de laquelle la lumière de l'ovéjecteur décrit un trajet spiralé complexe; à la suite du sphincter, l'ovéjecteur impair long de $360 \mu$ conserve des parois musculeuses épaisses.

Les deux utérus ont un trajet parfaitement rectiligne du fait qu'avec l'intestin ils emplissent totalement l'espace disponible dans la cavité générale. L'aspect du corps (fig. D) est donc très caractéristique, la maturation des œufs jusqu'aux microfilaires mûres s'effectuant régulièrement d'arrière en avant dans les deux tubes utérins parallèles. Les deux ovaires sont situés tout à l'arrière du corps. Les microfilaires mûres dans l'utérus sont longues de $130 \mu$. La queue est longue de $110 \mu$, la portion parenchymateuse de l'apex est légèrement lobée, alors que la cuticule qui la recouvre est lisse.

MaLE : Longueur de l'extrémité postérieure disponible : $31,5 \mathrm{~mm}$; largeur : $35 \mu$; Area rugosa constituée d'environ 45 bandes transversales de petits éléments bacilliformes (fig. F, G), étendues régulièrement sur une zone haute de $120 \mu$ qui débute à $12 \mu$ en avant du cloaque. Six paires de papilles cloacales disposées comme l'indique la figure F. Spicule gauche long de $270 \mu$, avec un manche de $160 \mu$ et une extrémité distale de forme caractéristique (fig. G). Spicule droit long de $105 \mu$, avec un manche de $70 \mu$ et une lame plus faiblement chitinoïde.

La queue est longue de $65 \mu$, les $20 \mu$ terminaux étant constitués d'un cône transparent qui donne l'impression d'être surajouté à l'extrémité postérieure, car il existe une autre assise cuticulaire arrondie, proche de l'extrémité parenchymateuse du corps (fig. F).

Microfilaire: Les frottis de sang contiennent quelques microfilaires assez mal colorées. Bien que plus courtes que les microfilaires utérines, nous pensons qu'il s'agit 


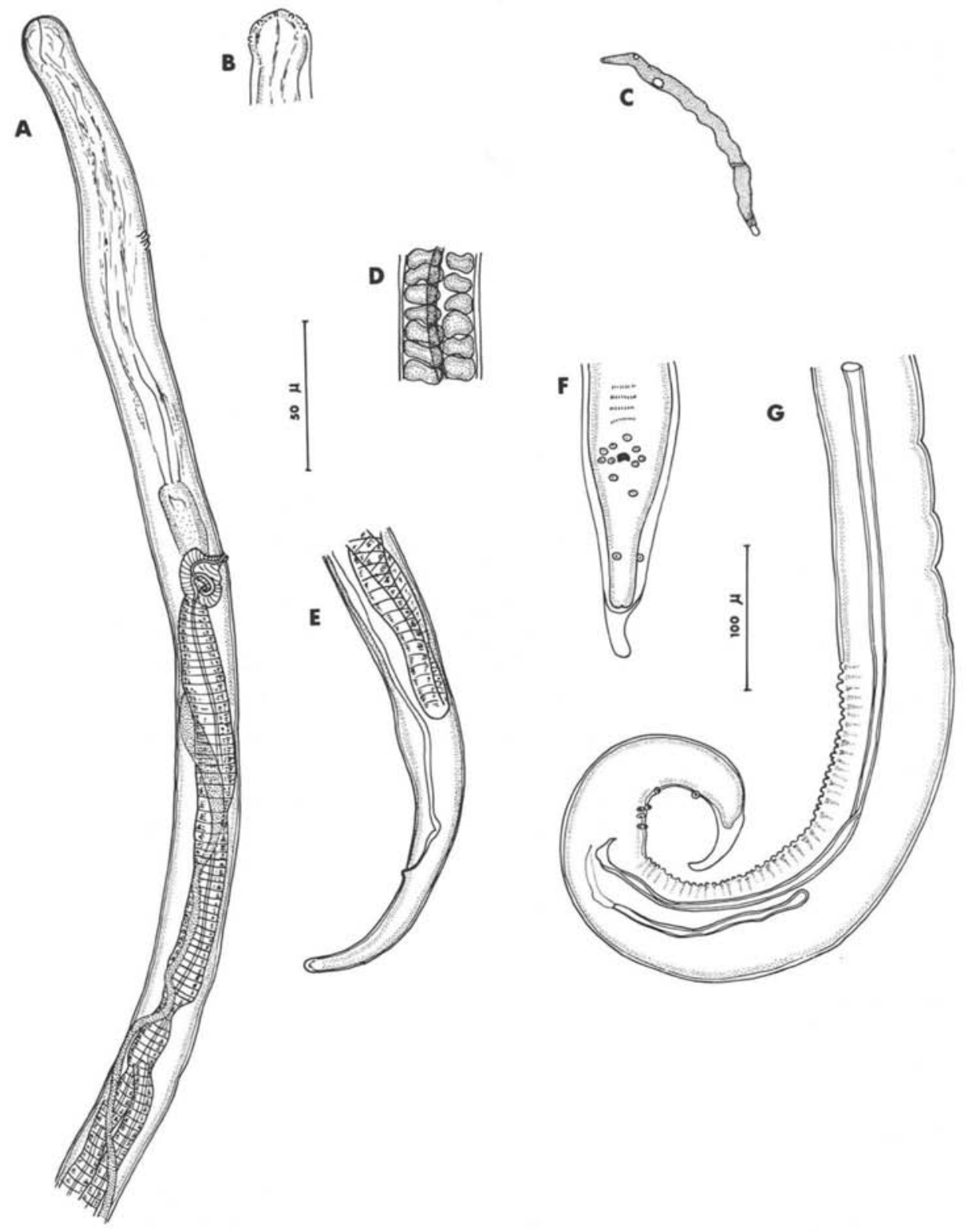

A : Extrémité antérieure, ㅇ, vue latérale; B: Extrémité céphalique comprimée, 우, vue latérale; C: Microfilaire sur frottis de sang; D: Partie moyenne du corps, $q$, les deux utérus et l'intestin occupent toute la place disponible ; E: Extrémité postérieure, q, vue latérale; F : Extrémité postérieure, ơ, vue ventrale; G : Extrémité postérieure, ơ, vue latérale, A, B, D, E: échelle $100 \mu ; \mathrm{C}, \mathrm{F}, \mathrm{G}$ : échelle $50 \mu$. 
de la même espèce, du fait qu'elles sont visiblement très rétractées sur le frottis. La microfilaire de Dipetalonema diacantha (Molin, 1858), également parasite de Coendu, est nettement plus longue (200 à $230 \mu$ selon Freitas et Lent, 1939).

Dépourvues de gaine, les microfilaires de nos frottis sont remarquables par une tête brusquement rétrécie (fig. C). Elles sont longues d'environ $90 \mu$ et larges de 4,5 $\mu$. L'anneau nerveux est à $23 \mu$ de l'apex, l'initiale mésenchymateuse $\left(R_{1}\right)$ à $23 \mu$ et le pore anal à $12 \mu$ de l'extrémité postérieure.

\section{Discussion.}

Comme l'attestent l'extrême gracilité du corps et l'œsophage atrophié, ces Filaires sont des formes hyperévoluées, ce qui les rend difficiles à classer.

L'élément morphologique le plus caractéristique est le cône transparent qui orne la queue du mâle. Cette formation a été observée sur un spécimen vivant et sur un autre spécimen fixé, et ne ressemble pas à une mue. Nous l'interprétons comme la fusion sur la ligne médiane de deux grands pétales caudaux de Dipetalonema.

En effet, l'extrémité caudale des mâles d'espèces telles que $D$. interstitium Price, 1962, D. llewellyni Price, 1962 ou D. perstans (Manson, 1891) redécrit par Chabaud (1952) évoque une évolution vers la création d'un cône terminal comparable à celui de notre espèce. Quelques espèces comme $D$. interstitium, D. llewellyni, $D$. obtusa (McCoy, 1936), redécrit par Esslinger (1966), ont un œsophage en voie d'atrophie et corrélativement, semble-t-il, un diamètre du corps très réduit.

Nous interprétons donc la nouvelle espèce du Coendu comme une hyperévolution des espèces citées plus haut et sommes tentés de la placer dans le genre Dipetalonema. Cependant, d'un point de vue théorique, l'interprétation que nous donnons du cône transparent de la pointe caudale du mâle, caractéristique de l'espèce, reste hypothétique ; d'un point de vue pratique, la définition du genre Dipetalonema devient très difficile à formuler si l'on y incorpore des espèces à œsophage très atrophié et n'ayant aucun pétale caudal.

Nous pensons donc qu'il est plus simple de créer un genre nouveau pour cette espèce, et nous proposons le nom de Filarissima lainsoni n. gen., n. sp., pour la dédier à notre collègue et ami le $\mathrm{D}^{r}$ Ralph Lainson, qui avec une extrême gentillesse a facilité nos recherches helminthologiques à Belem.

\section{Définition.}

Filarissima n. gén. Onchocercinae avec diamètre du corps très réduit, ne laissant de place que pour deux tubes utérins et l'intestin. Esophage atrophié. Vulve un peu en arrière de la fin de l'œsophage. Queue du mâle terminée par un grand appendice conique transparent.

Espèce type: $F$. lainsoni n. sp. 


\section{Bibliographie}

Chabaud (A.-G.), 1952. - Le genre Dipetalonema Diesing, 1861 ; essai de classification. Ann. Parasit. hum. comp., 27, 250-285.

Esslinger (J.H.), 1966. - Dipetalonema obtusa (Mc Coy, 1936) comb. n. (Filarioidea: Onchocercidae) in Colombian Primates, with a description of the adult. J. Parasit., $52,498-502$.

Freitas (J.F. Teixeira de) et Lent (H.), 1939. - Novo genero de helmintos parasitos de roedores. (Nematoda : Filarioidea). Boletim Biol. (Nova série), 4, 14-18.

PrICE (D. L.), 1962. - Description of Dipetalonema interstitium n.sp. from the Grey Squirrel and Dipetalonema llewellyni n. sp. from the Raccoon. Proc. Helm. soc. Wash., 29, 77-82. 